\title{
Semi-automated Assembly of a MEMS-Based Micro-scale CMM Probe and Future Optimization of the Process Chain with a View to Desktop Factory Automation
}

\author{
James D. Claverley ${ }^{1}$, Arne Burisch ${ }^{2}$, Richard K. Leach ${ }^{1}$, and Annika Raatz ${ }^{2}$ \\ ${ }^{1}$ Engineering Measurement Division, National Physical Laboratory \\ Hampton Road, Teddington, UK, TW11 0LW \\ james.claverley@npl.co.uk \\ ${ }^{2}$ Institute of Machine Tools and Production Technology (IWF), \\ Technische Universität Braunschweig, Braunschweig, DE \\ a.burisch@tu-bs.de
}

\begin{abstract}
The assembly process of a novel micro-scale co-ordinate measuring machine probe is presented. The process makes use of a semi-automated miniature robot. The initial tests that led to the full process chain are described, and the full process chain presented. The presented process chain successfully produced four assembled probes. Future work is suggested to augment the presented process chain leading to further automation.
\end{abstract}

Keywords: micro-CMM probe, MEMS, micro-assembly, desktop factory.

\section{Introduction}

Modern precision engineering demands measurement capabilities of at least equal, if not superior, accuracy to the machining capabilities. This statement is especially true for small components with functional micro-scale features. The required measurement capability is partially realized by the new generation of micro-co-ordinate measuring machines (micro-CMMs), such as the Zeiss F25, however, existing high accuracy probing systems for such machines often lack all the functionality required to complete such measurements [1].

The National Physical Laboratory (NPL) has developed a novel micro-scale CMM probe that aims to bring overall measurement uncertainty of micro-CMMs to below $100 \mathrm{~nm}$, while also allowing true isotropic 3D scanning and non-contact surface detection. The probe consists of a triskelion (three-legged) nickel flexure device and a spherical stylus tip.

The triskelion device is a MEMS (micro-electrical mechanical system) produced using typical integrated circuit production techniques such as metal deposition and chemical etching [2]. A $7 \mu \mathrm{m}$ thick piezo-electric (lead zirconium titanate) film is deposited onto the nickel flexures using a sol-gel spinning technique. Each flexure is $2 \mathrm{~mm}$ long, $0.2 \mathrm{~mm}$ wide and nominally $20 \mu \mathrm{m}$ thick. The flexures are fabricated on a silicon chip, $14 \mathrm{~mm}$ by $14 \mathrm{~mm}$ by $1 \mathrm{~mm}$. 
The spherical tipped stylus is $2 \mathrm{~mm}$ long, $200 \mu \mathrm{m}$ in diameter with a $70 \mu \mathrm{m}$ diameter spherical tip. It is produced using micro-electro-discharge machining. A full description of the production process can be found elsewhere [3], [4].

Using the deposited PZT as actuators and sensors, the device is designed to vibrate in a direction normal to any measurement surface it encounters. This vibration is used to counteract the disruptive surface forces encountered during micro-scale dimensional measurement and to enable the probe to operate in a non-contact mode, significantly reducing the probing force imparted on the measurement surface in comparison to existing micro-CMM probes [5]. A schema of the device is shown in figure 1.

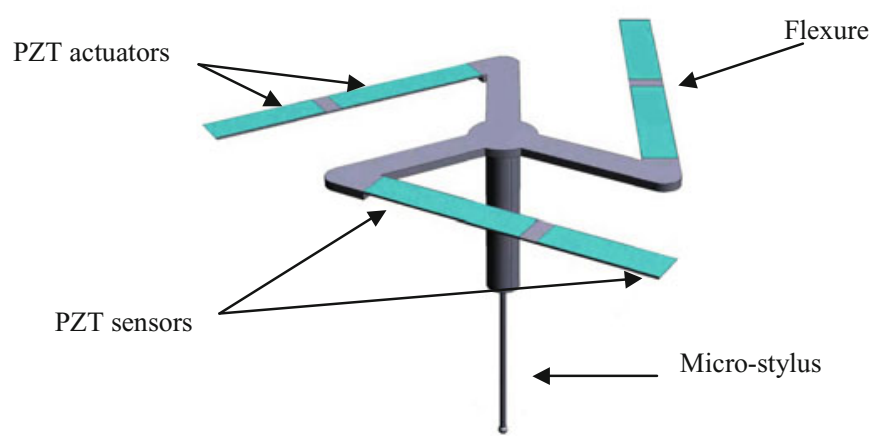

Fig. 1. A schema of the vibrating micro-CMM probe

The assembly of the flexure chip and the sphere-tipped stylus, to produce the vibrating micro-CMM probe, presents a number of challenges. These include, but are not limited to, the delicate nature of the $20 \mu \mathrm{m}$ nickel thick flexures, the difficulty in handling millimetre-sized parts, the surface interaction forces being dominant over gravity during the operation and obscured view of critical process steps due to limited available camera positions. The ideal physical requirements of the assembly of the stylus onto the chip are a positional accuracy of $\pm 0.5 \mu \mathrm{m}$ and an angle between the stylus and flexure of $90^{\circ} \pm 0.29^{\circ}[6]$.

\section{Assembly Preparation and Tests}

There are many demands put on processes for micro-scale assembly. These demands include the need for high precision and repeatability, flexibility and energy efficiency, low cost and suitable workspace volume and shape. Most conventional assembly machines do not guarentee all of these requirements to the level demanded by this product along with a high degree of automation [7]. New approaches for scalable and highly flexible production technology are miniaturized handling devices in the range of several centimetres to a few decimetres [8].

\subsection{Assembly Setup}

To complete the assembly of the flexure and the spherical stylus tip, a miniaturized assembly setup using a robot (Parvus) was developed at the Institute of Machine 
Tools and Production Technology (IWF) of TU Braunschweig. A micro-gripper [9] was connected to the robot and two microscope cameras were arranged within the workspace with front and lateral views. A fixture was designed and manufactured to hold the triskelion chip on a tip-tilt table within the robot's workspace. An ultraviolet (UV) light source was also arranged within the workspace. The capability of this setup beyond that of conventional assembly systems, including accuracy, repeatability and the scope for automation, is fully described in [3]. The setup is shown in figure 2.

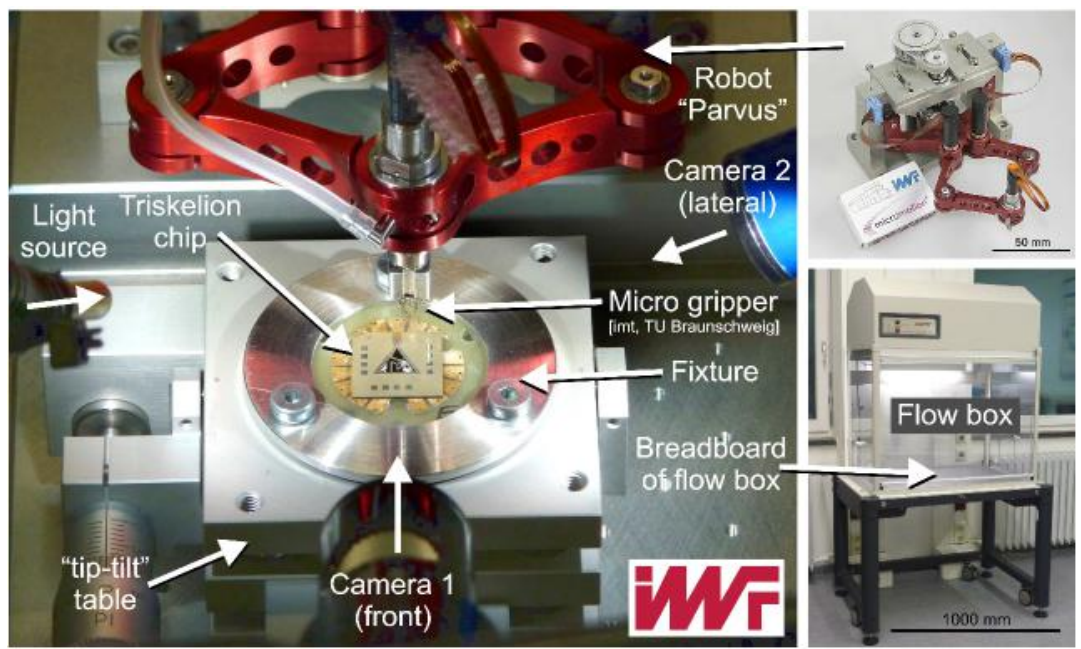

Fig. 2. Setup of the assembly system, robot Parvus and flow box

\subsection{Camera Verification}

The cameras are used during the assembly process to verify the angle between the triskelion and the stylus. Both cameras were verified using a previously calibrated vertical micro-scale artefact. This artefact was positioned directly above the centre of the triskelion device. The camera was thus calibrated to the upper surface of the triskelion device.

\subsection{Experiment with UV Curing Adhesive}

Ultraviolet curable adhesive was chosen as the most suitable adhesive for the assembly [3]. Several types of UV curable adhesive were investigated specifically focusing on their uncured viscosities. These are shown in table 1.

Table 1. Physical properties of several adhesives tested for their suitability [10, 11, 12, 13]

\begin{tabular}{lll} 
Adhesive & Viscosity /mPa & Hardening at wavelength /nm \\
\hline DELO Katiobond AD 610 & 400 & $320-380$ \\
DELO Katiobond 4578 & 12400 & $400-550$ \\
Vitralit 1605 & 500 & $400-500$ \\
Loctite 352 & 20000 & 365 \\
\hline
\end{tabular}


Adhesive types DELO Kationbond 4578 and Vitralit 1605 cure under normal white light after a few minutes and are thus not suitable for the assembly process, which may take some minutes. For the adhesive with the lowest viscosity (DELO Katiobond AD610), dipping and application with a hollow dispenser needle (diameter $0.24 \mathrm{~mm}$ ) deposited an adhesive drop onto a test surface with a diameter of $320 \mathrm{jtm}$. A similar test using the high viscosity adhesive (Loctite 352) resulted in drops with diameters in the range of $210 \mathrm{jtm}$ to $240 \mathrm{jtm}$. The aim of the investigation was to select the adhesive that deposited the smallest uncured drop, therefore Loctite 352 was chosen for the assembly process.

A Hartmann UV-P 280/2 light source was used to cure the adhesive. After a curing time of $60 \mathrm{~s}$ the Loctite 352 adhesive drop was tack free.

\subsection{Application of Adhesive to Triskelion}

To complete adhesive tests on an actual triskelion, a dispenser needle (diameter 0.24 $\mathrm{mm}$ ) was dipped into an adhesive reservoir. A drop was applied on the central area of the triskelion as shown in figure 3.
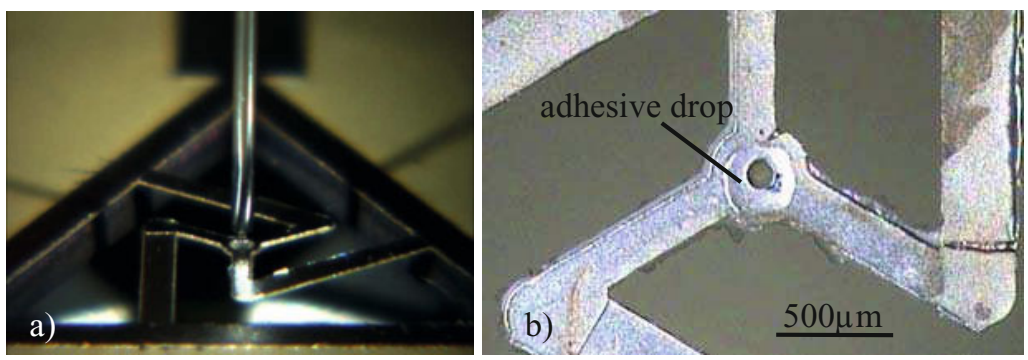

Fig. 3. A small adhesive drop being deposited (a) in the centre of the triskelion. The resulting adhesive drop is shown at (b).

Dipping the dispenser needle into the adhesive reservoir worked well in the first experiments on a test surface (see section 2.3). However, this method does not apply an exact or repeatable amount of adhesive. To solve this problem, the Parvus robot was equipped with a dispenser needle that can directly apply a pre-defined volume of adhesive on to the surface of the triskelion. Additionally, to ensure sufficient contact between the triskelion surface, the stylus and the adhesive, the stylus was also dipped into the adhesive reservoir, as shown in figure 4.
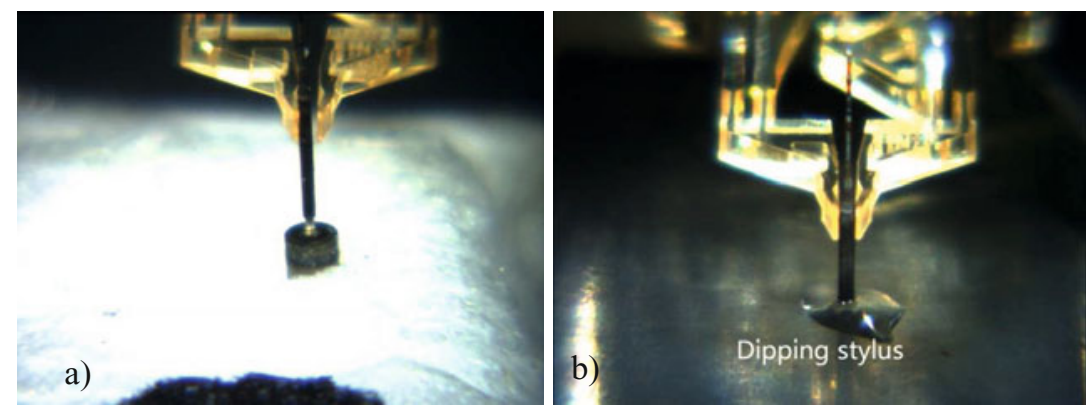

Fig. 4. Gripping the stylus (a) and dipping the end in an adhesive reservoir (b) 


\subsection{Test Assembly of Stylus}

Once the assembly setup was built, the cameras verified and a gluing strategy devised, it was necessary to complete a trial manual assembly.

Firstly, a micro-stylus was selected and prepared by removing it from its transport stub and inspecting it under a 3D microscope. The transport stub is designed to allow easy transport and handling of the stylus without gripping the delicate stylus directly. The design has been suitable to allow transport of the styli from Taiwan to the UK via normal commercial courier. It was possible to grip the stylus on its transport stub with the micro-gripper, however, the necessary force to break the stylus at the neck is too high and experience suggested that the gripper would break. Hence, the stylus was removed manually and placed safely in a gel receptacle, where the gripper could collect it. This process is shown in Figure 5.
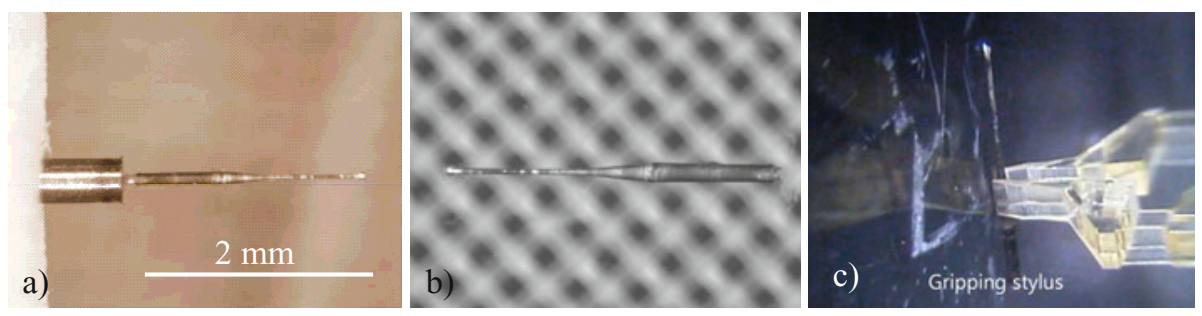

Fig. 5. Micro-stylus in transport stub (a), being inspected (b) and collected for assembly (c)

Once both adhesive depositing steps were completed, as described in section 2.4, the stylus was positioned over the central area of the triskelion and moved down into contact with it. Finally, the adhesive was cured, fixing the stylus in place as shown in figure 6 . To check the stability of the connection between the triskelion and the stylus, a lateral force was applied to the stylus using the micro-gripper, also shown in figure $6 b$.
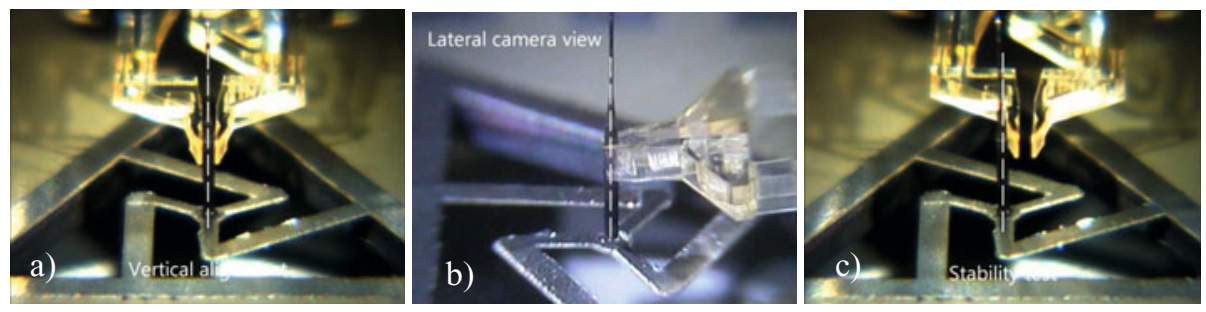

Fig. 6. The completed test assembly, front view (a), lateral view (b). A stability test is also completed on the stylus (c).

\section{Final Assembly Process}

Working from all previous experiments and test assemblies, as described in section 2, a full process chain was devised to assemble the micro-probe. This process chain was 
developed to allow the maximum amount of preparation before any irreversible steps are conducted. The preparation steps include aligning the fixture within the robot's workspace and mounting the various adhesive applicators. The irreversible steps include depositing adhesive on the parts, bringing those parts together in assembly and curing the adhesive. These steps should only be completed once all preparation has been performed and it is possible to complete assembly without interruption or breaks.

At several stages throughout the assembly process, the Parvus robot was taught the locations of several essential positions within the workspace. This allows the operator to take advantage of as much automation as it was possible to devise in the short time frame of the project. The complete process chain is shown in table 2.

Table 2. Complete process chain for assembly of triskelion and stylus, including links to previous figures for reference; the irreversible steps are indicated with an asterisk (*)

Process description $\quad$ Reference

1 Verify condition of stylus and triskelion under 3D microscope

Figure 5

2 Mount triskelion device and fixture on the tip-tilt table

Figure 2

3 Insert plate for adhesive dipping

Figure $4 b$

4 Align tip-tilt table in the workspace of the robot

Figure 2

5 Position the calibrated part for camera calibrationSection 2.2

6 Arrange cameras (front and lateral) and approx. $30^{\circ}$ from horizontal

- Align vertical orientation of cameras according to the calibration part

7 Teach robot rough position of gripper over the central hole of triskelion

8 Teach robot rough position of gripper over the plate for adhesive dipping

9 Mount adhesive cartridge with dispenser needle onto robot

10 Teach robot rough position of adhesive dispenser over central hole of triskelion

11 Remove adhesive cartridge

12 Remove tip-tilt table

13 Position gel-tray with stylus within the robot workspace

14 Grip the stylus with the micro gripper

Figure 5c

- If necessary correct stylus' position within the gripper

15 Remove gel-tray

16 Re-insert tip-tilt table

17 Test assembly without adhesive

- Test of process 7, re-teach assembly position if necessary

18 Mount adhesive cartridge

19 Check pressure and dispensing time at adhesive dispenser

20 Dispense adhesive drop on plate

Figure $4 b$

$21^{*}$ Dispense adhesive drop on the centre of triskelion

Figure 3

22 Remove adhesive cartridge

23* Dip stylus in the adhesive drop on the plate

Figure $4 b$

24* Assemble the stylus onto the triskelion

Figure 6a,b

25 Align stylus vertically

Figure 6a,b

26* Cure adhesive by ultraviolet light

27 Open the gripper and retract 


\section{$4 \quad$ Results and Conclusion}

Four micro-CMM probes were successfully assembled using the method described. The assembly process, as described in table 2, was 30 minutes in duration per probe. The time consuming factor is the lack of adequate feeding systems for micro parts.

With automated feeding, the assembly could be completed in 5 minutes. Each completed micro-CMM probe was qualitatively tested to establish the orthogonality of the stylus. Each completed probe was also functionally tested by applying a lateral force on the stylus to verify sound adhesion between the assembled parts.

The assembly method uses automation to ensure repeatable volumes of adhesive are deposited on the triskelion and to ensure repeatable placing of the stylus at the triskelion. However, many operations, such as picking up and handling of the stylus, and fine adjustments of the position of the stylus before curing are done under manual control. As a prototype assembly method, processing small volumes of parts, this semi-automation is sufficient. However, should there be a need to scale up the process for higher production volumes, more automation would have to be implemented.

\section{$5 \quad$ Future Work}

With a view to increasing the automation of the assembly process, several steps can be taken to make robot handling and programming easier.

The stylus transport stub should be redesigned so that the micro-gripper can safely remove the stylus without the need for a manual operation and an intermediate receptacle. The styli should also be supplied in cartridges, which will allow for programmable gripping by the micro-gripper. Furthermore, automated feeding of the styli will be necessary to guarantee a continuous assembly process.

The triskelion device should be automatically transported to the assembly position. The triskelion device could directly be gripped and positioned by the Parvus with a typical vacuum gripper. Therefore, a tool changer, which is currently under development at the IWF, could be used to switch between the vacuum and the microgripper. Alternatively, an additional feeding system for the triskelion devices could bring the parts into the assembly position. Furthermore, an active fixture, as presented elsewhere [8], that clamps and releases the triskelion devices will be necessary.

The current camera positions (front and side, elevated at about $30^{\circ}$ ) are ideal for observing and controlling the adhesive deposition steps and also any stylus manipulation steps. However, they do not allow for quantitative measurement of stylus angle. They are also susceptible to any rotational misalignment on setup and misalignment of reference features on the triskelion. A variable focus camera should be set up directly above the centre of the triskelion to allow for better measurement of the stylus angle and to verify that the sphere tip is directly above the centre of the triskelion. While the process could, theoretically, be run using only this camera, any imperfections in the parts (straightness or flatness errors) will cause errors in the assembly. For adequate control, at least two cameras are required, for ideal control, three cameras should be used.

The position of the assembly point, at the centre of the triskelion, should be determined prior to assembly. If the assembly point can be determined with respect to the origin of the Parvus robot, the operator will not need to manually teach the assembly point. Alternatively, a vision system and automated image processing or a laser scanning process could determine the assembly position. 
All parts, fixtures and cartridges should be redesigned so that they can fit easilyinside the workspace. This will reduce the need to remove and replace parts during the assembly process.

A set of dummy parts should be made to allow these changes to be tested. Using these dummy parts a higher production scale can be simulated without the waste of expensive micro-parts.

Acknowledgments. This work was funded by the UK National Measurement Office Engineering and Flow Metrology Programme 2008 to 2011. The authors would like to thank Christopher Jones (NPL), Prof. Dong-Yea Sheu (NTUT) who developed the probe production techniques [4] and the Microsystems and Nanotechnology Centre (Cranfield University) for producing the triskelion devices.

The assembly setup installed at IWF used a micro gripper and further knowledge developed within the Collaborative Research Centre 516, funded by the German Research Foundation.

\section{References}

1. Leach, R.K.: Fundamental principles of engineering nanometrology. Elsevier (2009)

2. Stoyanov, S., Bailey, C., Leach, R.K., Hughes, B., Wilson, A., O'Neill, W., Dorey, R.A., Shaw, C., Underhill, D., Almond, H.J.: Modeling and prototyping the conceptual design of 3D CMM micro-probe. In: Proc. 2nd ESITC, Greenwich, September 1-4, pp. 193-198 (2008)

3. Claverley, J.D., Sheu, D.-Y., Burisch, A., Leach, R.K., Raatz, A.: Assembly of a novel MEMSbased 3D vibrating micro-scale co-ordinate measuring machine probe using desktop factory automation. In: Proc. IEEE ISAM, Tampere, FI, May 25-27 (2011) ISBN 9781612843414

4. Sheu, D.-Y.: Micro spherical probe machining by EDM. J. Micromech. Microeng. 15, 185-189 (2005)

5. Claverley, J.D., Leach, R.K.: A vibrating micro-scale CMM probe for measuring high aspect ratio structures. Microsyst. Technol. 16, 1507-1512 (2010)

6. Smale, D., Ratchev, S., Segal, J., Leach, R.K., Claverley, J.D.: Assembly of the stem and tip of an innovative micro-CMM probe. In: Euspen 9th Int. LAMDAMAP, Uxbridge, UK, June 29-July 2, pp. 442-451 (2009)

7. Burisch, A., Soetebier, S., Wrege, J., Sattler, R.: Design of a parallel hybrid micro-scara robot for high precision assembly. Mechatronics and Robotics 4, 1374 (2004)

8. Burisch, A., Wrege, J., Raatz, A., Hesselbach, J., Degen, R.: PARVUS - Miniaturized robot for improved flexibility in micro production. J. Assembly Automation 27(1), 65-73 (2007)

9. Hoxhold, B., Wrege, J., Bütefisch, S., Burisch, A., Raatz, A., Hesselbach, J., Büttgenbach, S.: Tools for Handling and Assembling of Microparts. In: Büttgenbach, S., Burisch, A., Hesselbach, J. (eds.) Design and Manufacturing of Active Microsystems. Microtechnology and MEMS series, pp. 393-409. Springer, Berlin (2011)

10. DELO Industrie Klebstoffe: Product manual (DELO Katiobond AD610) Revision 27, pp. 2-5 (2010), http: / /www. delo.de/fileadmin/datasheet/

DELO-KATIOBOND_AD610_(TIDB-GB) .pdf (accessed June 29, 2011)

11. DELO Industrie Klebstoffe: Product manual (DELO Katiobond 4578) Revision 26, pp. $2-$ 5 (2010) http: / /www.delo.de/fileadmin/datasheet/

DELO-KATIOBOND_4578_(TIDB-GB) .pdf (accessed June 29, 2011)

12. Etilux: Product manual (Vitralit 1605) Revision: 03/2006, p. 3 (2006), http: / /www. etilux.com/medias/catalog/multimedia/ DocVtralitrev03-06.pdf (accessed June 29, 2011)

13. Henkel: Product manual (Loctite 352) Revision: January 2009, pp. 1-2 (2009), http: //tas. loctite. com/tds5/docs /352-EN. pdf (accessed June 29, 2011) 